\title{
Rutas pedagógicas en la formación de licenciados en matemáticas: dificultades para su transformación ${ }^{1}$
}

\author{
Luisa Andrade* \\ Claudia Salazar* \\ Cecilia Leguizamón*
}

Artículo recibido: 12-5-2005 y aprobado: 2-5-2007

\begin{abstract}
Pedagogical paths in a mathematics teacher training program: difficulties in transforming them
\end{abstract}

Resumen: Al observar cuatro cursos de un programa de licenciatura en matemáticas, se encuentra que tanto los principios curriculares del programa como las intenciones de los profesores para las rutas pedagógicas están permeados por planteamientos innovadores, en especial por ideas concernientes a construir conocimiento como producto de la interacción social, a promover la formación de ciudadanos críticos y comprometidos, a aproximarse a los conceptos matemáticos desde diferentes ángulos. Si bien se evidencia incorporación cultural de estas ideas a nivel del vocabulario de profesores y estudiantes, con el uso de nuevos términos y expresiones, las rutas que al respecto se siguen en clase son bastante vagas y reflejan las enormes dificultades que hay para la concreción de tales constructos teóricos.

Palabras claves: educación matemática, didáctica de las matemáticas, formación de formadores, práctica docente, investigación cualitativa.
Summary:Once having observed four courses of a mathematics teacher training program, we found out that both, curricular principles and teacher intentions for pedagogical paths are permeated by innovative formulations, particulary by ideas concerned with the construction of knowledge as a result of social interaction, the promotion of critical and committed citizens, and the approach of mathematical concepts from different perspectives. Although there are signs of cultural incorporation of these ideas in the vocabulary of teachers and students, with the use of new words and expressions, the related paths followed in class are vague and show the difficulties involved in implementing those theoretical constructs.

Key words: mathematics education, didactics of mathematics, mathematics teacher training, mathematics teaching, qualitative investigation

1 Este estudio se llevó a cabo en el marco del Nuevo Proyecto Curricular de la Licenciatura en Matemáticas de la Universidad Pedagógica Nacional de Bogotá, en el año 2004 y contó con el apoyo financiero del centro de investigaciones de la Universidad, CIUP.

* Profesores del Departamento de Matemáticas de la Universidad Pedagógica Nacional. 


\section{Introducción}

Las concepciones que fundamentan la formación de educadores matemáticos, y por consiguiente los programas encaminados a tal fin, han experimentado recientemente cambios profundos, propiciados por la consolidación de la educación matemática como campo disciplinar e investigativo que se ocupa tanto de los objetos del conocimiento matemático y de los procesos de enseñanza, aprendizaje y evaluación en matemáticas, como de las condiciones sociales y culturales que los afectan. Los nuevos referentes conceptuales describen al docente como promotor y actor del cambio con una participación activa en el diseño y, por supuesto, en el desarrollo curricular, e indican que un programa de formación de docentes que pretenda atender a las exigencias del entorno educativo actual y contribuir a mediano, o largo, plazo a la mejora sustancial de la calidad del aprendizaje de los estudiantes, debe aportar a desarrollar aspectos del profesor de los que depende su práctica (Andrade y Perry, 2002).

A partir de las consideraciones anteriores y de las acciones investigativas de los profesores del Departamento de Matemáticas de la Universidad Pedagógica Nacional, se crea en 1999 el Nuevo Proyecto Curricular de la Licenciatura en Matemáticas, en el que se plasman las condiciones necesarias para la formación del profesional de la educación matemática del país. Un elemento indispensable de cualquier desarrollo curricular que realimenta y redirige el proceso, es la evaluación del mismo. Para el caso particular de este Proyecto se hace necesario el seguimiento sistemático a su implementación que permita comprender los procesos de innovación curricular que allí se llevan a cabo, y determinar la correspondencia entre la realidad de su ejecución y los planteamientos propuestos en los documentos que lo definen, y aportar así a su actualización y posibles transformaciones.

Se adelanta entonces, durante 2004, un estudio cuyo propósito es develar las rutas pedagógicas adoptadas en la formación de licenciados en matemáticas. Las rutas pedagógicas se han conceptualizado por el Instituto para la investigación Educativa y el Desarrollo Pedagógico, Idep (Beltrán, Pardo y Cruz, 2003) como el conjunto de elementos involucrados en un desarrollo currícular, relacionados con el nivel institucional, la metodología, la didáctica, el conocimiento. Los aspectos relativos a la metodología y la didáctica de las rutas tienen que ver con normas prescriptivas o saberes regulados por una planeación y parámetros que el maestro establece. Por tanto, en toda ruta pedagógica se encuentran tanto la práctica docente del maestro, entendida como una forma de saber pedagógico, como el aula de clase, concebida como el campo donde se desarrollan diversas estructuras que determinan y limitan los acontecimientos pedagógicos.

En la misma dirección, Andrade, Perry, Guacaneme y Fernández (2003a) señalan que en la construcción y puesta en práctica de rutas pedagógicas, el actor principal es, sin duda, el profesor de matemáticas; es él quien de manera natural, legítima y directa tiene la autonomía y las oportunidades para buscar e implementar alternativas de solución que propendan por unos resultados eficaces de su quehacer profesional en la formación de los estudiantes.

En este estudio se analizan las rutas pedagógicas a través del exámen de los 
documentos que trazan tales rutas y de lo que ocurre en el salón de clase, en especial de la práctica docente del profesor como la expresión primordial de las rutas pedagógicas implementadas. Dado que esta mirada arroja información sobre innumerables aspectos explícitos de la clase, y posiblemente sobre otros tantos tácitos, se impone la necesidad de delimitar el foco del estudio de manera que se pueda profundizar en la exploración y reflexión de asuntos concretos. En consecuencia, de los muchos aspectos que pueden constituir una ruta pedagógica, se consideran algunos que emergen como centrales a la innovación propuesta en la Licenciatura en Matemáticas, y que se perciben como peculiares en algunas de las observaciones de clase. La conceptualización de estos aspectos constituye el marco construido, en concordancia con la postura de Andrade, Perry, Guacaneme y Fernández (2003a), quienes afirman que "al caracterizar un fenómeno tan complejo como la enseñanza de las matemáticas, resulta imprescindible elaborar un marco conceptual que permita dar cuenta de cómo hace el profesor lo que hace".

En este artículo se presenta una síntesis del estudio realizado. En primer lugar se da cuenta de apartes de la metodología empleada y del marco conceptual elaborado para la recolección y análisis de los datos. En seguida, se expone una discusión de algunos de los resultados encontrados y unas anotaciones finales.

\section{Aspectos metodológicos de la investigación}

De acuerdo con Denzin y Lincoln (1998), la investigación cualitativa estudia las cosas en su entorno natural, intentando darle sentido o interpretar los fenómenos en términos de los significados que las personas le dan, por tanto involucra, como señala Quinn (2002), una aproximación interpretativa y naturalista al asunto en cuestión, que propende por reconstruir las experiencias de la actividad humana (Eisenhart, 1988). Además, según estos mismos autores, los investigadores cualitativos emplean un amplio rango de métodos moldeados por la misma investigación y una diversidad de fuentes de información interconectadas: la observación directa, las entrevistas de profundización, los documentos escritos, etc., con la esperanza siempre de obtener una mejor comprensión del asunto. Este estudio, tanto por el objeto y los propósitos, como por la diversidad de los instrumentos de recolección de datos que se han usado, se inscribe en la corriente de investigación cualitativa. El objeto a estudiar son las rutas pedagógicas determinadas prioritariamente por la práctica docente en clase del profesor de la Licenciatura en Matemáticas, como una experiencia social a la que se pretende dar sentido en la formación de licenciados, enfoque con el que la investigación cualitativa considera toda actividad humana (Eisenhart, 1988).

La selección de los espacios académicos a observar se debió a la disponibilidad y aceptación de los profesores encargados de los cursos, y la cantidad se determinó de acuerdo con las posibilidades, recursos y alcances del estudio. Dado que la observación se centró en cuatro espacios académicos, no se pretende afirmar que los resultados puedan generalizarse a todos los cursos de la Licenciatura, pero sí se cree, coincidiendo 
con la perspectiva de estudios de caso de la investigación cualitativa, que una examinación detenida y muy próxima de cuatro casos, puede ser más reveladora que una indagación cuantitativa con una muestra extensa. En este estudio fue posible identificar acciones, relaciones e intervenciones puntuales de los participantes, que proveen información que de otra forma seguramente no se hubieran encontrado.

Tres actividades fundamentales, desarrolladas de manera paralela, constituyeron el trabajo primordial del equipo de investigación a lo largo del estudio. En primer lugar, la recolección de información proveniente de diversas fuentes como: los programas de cada espacio académico elaborados por los profesores; las observaciones no participantes de clase, consignadas en notas de campo y en grabaciones de audio y video; las guías y talleres propuestos por los profesores; las tareas y lecturas asignadas a los estudiantes y las entrevistas semiestructuradas que se llevaron a cabo con los profesores responsables de los espacios académicos involucrados y con tres estudiantes de cada uno de dichos cursos. En segundo y tercer lugar, la elaboración del marco conceptual para develar las rutas pedagógicas y el análisis e interpretación de los datos, respectivamente.

La lectura de documentos, la revisión de la información recopilada, el trabajo individual de clasificación, la reflexión colectiva, fueron las acciones de un proceso cíclico que definió las categorías de este marco. Así, los datos recogidos se examinaban, comparaban y verificaban para detectar aspectos comunes a los espacios académicos y características importantes de las rutas pedagógicas seguidas, que daban pie a la conformación de las categorías. Las diferencias encontradas entre datos de una misma categoría obligaron a precisar y limitar las categorías, a establecer criterios cada vez más concisos para la clasificación y a revisar de nuevo los datos. En este proceso las categorías definitivas, sufrieron numerosas modificaciones y ajustes, atendiendo además a la consideración de los planteamientos del Nuevo Proyecto Curricular y del deber ser de la Licenciatura en Matemáticas (Departamento de Matemáticas-UPN, 2002) y a la identificación de posibles resultados del estudio que pudieran ser de interés para el Departamento.

En este estudio, con el fin de añadir profundidad y rigor a los resultados que se proclaman, se empleó la triangulación como alternativa a la validación, la cual implica la contrastación de los resultados entre varias personas, desde distintas perspectivas y en diversas situaciones; dicha contrastación se vivenció a través de la dinámica misma de trabajo individual y colaborativo que se dio entre los participantes, de la información recopilada por diferentes medios que permitió comparar y verificar interpretaciones, de la lectura comentada de los documentos elaborados por parte de los profesores observados y de algunas personas externas a la investigación.

\section{La conceptualización elaborada}

El marco conceptual elaborado define y delimita en cinco categorías los aspectos contemplados. Estas categorías permiten no sólo organizar y analizar la información, sino además especializar la mirada en la observación de las clases. 
Se considera así una categoría de metodología que contempla las acciones que tanto el profesor como los estudiantes llevan a cabo en clase, y el tipo de tareas que se proponen a los estudiantes desde un punto de vista metodológico general, no necesariamente enlazado con los contenidos tratados en un curso determinado. Otra categoría más específica apunta a las situaciones donde se da interacción entre los asistentes a clase en torno a los dominios de estudio, y se denomina construcción social del conocimiento. Una tercera categoría agrupa posibles incidencias de lo que sucede en clase, en la formación de los estudiantes como ciudadanos y futuros maestros. La cuarta categoría abarca información relativa a las estrategias de evaluación que se ponen en juego para la promoción de los estudiantes. Finalmente se trabaja una categoría acerca del contenido disciplinar abordado en los cursos y su desarrollo.

\section{Metodología}

El profesor es el responsable de concebir y llevar cabo los planes de formación de los estudiantes, cualesquiera que sean tales planes e independientemente del tipo de acciones y tareas que los compongan. Existe entonces, como lo expresa Rico (1990), la convicción de que cada profesor debe disponer de un esquema global de cómo actuar en el aula, que incluya las fases que deben cubrirse en la actuación, los métodos que se seguirán para enseñar, el tipo de tareas que deben realizar los estudiantes, los recursos que deben utilizar, las conductas que se quieren estimular y conseguir. Este esquema es lo que usualmente se conoce como metodo- logía, término que ha designado desde tiempos atrás la forma grosso modo en que un profesor desarrolla sus clases. También hay un consenso ampliamente compartido de que no existe un método que pueda considerarse como el ideal para enseñar matemáticas, pero a pesar de esto, "los profesores desearían que se les señalase el método más idóneo para enseñar matemáticas; sin embargo, esto no es posible ni deseable" (Cockcroft, 1985).

La noción de currículo más difundida contempla la metodología como uno de sus aspectos y para Rico (1997) éste es uno de los cuatro componentes que caracterizan el currículo como plan operativo de actuación del profesor. Diferentes autores y propuestas han sugerido lineamientos metodológicos a seguir, junto con sus bondades, que se refieren a las acciones del profesor, los tipos de tareas en los que se deben involucrar los estudiantes, los recursos que se deben emplear, el papel del profesor y de los estudiantes. En la formulación del Nuevo Proyecto Curricular de la Licenciatura en Matemáticas de la Universidad Pedagógica Nacional (Departamento de Matemáticas-UPN, 2002), se indica que las prácticas educativas deben ser una oportunidad para que el futuro profesional de la educación reflexione sobre la enseñanza y el aprendizaje, desarrolle conocimiento didáctico y se acerque a la investigación en el aula. Esto es apoyado por Bonilla y Romero (2000) quienes afirman que en la formación de maestros se requiere transformar las concepciones y creencias que traen los estudiantes acerca de las matemáticas, la enseñanza y el aprendizaje, generadas por su experiencia como estudiantes, a 
través de ambientes de aprendizaje que les permitan aprender de la manera en que se espera que puedan enseñar.

En este estudio para esta categoría se identifica la actuación del profesor y de los estudiantes en el desarrollo de las clases de manera que permita dar cuenta de la metodología empleada. Las actividades en esta categoría se observan básicamente desde un ángulo relacionado con la función de manejo del curso en el sentido propuesto por Doyle (1986), es decir, como parte de un proceso que tiene una estructura y un propósito, más que desde la perspectiva de la actividad matemática implicada en su desarrollo, la cual se considera en la categoría del contenido.

Tabla 1. Metodología.

\begin{tabular}{|l|l|}
\hline \multirow{4}{*}{$\begin{array}{l}\text { Actuación } \\
\text { del } \\
\text { profesor }\end{array}$} & $\begin{array}{l}\text { Acciones como exposición de temas, ilustración del desarrollo de ejercicios, asigna- } \\
\text { ción de tareas, preguntas a los estudiantes, cordinación de discusiones, realización de } \\
\text { evaluaciones, presentación de información para el funcionamiento del curso, revisión } \\
\text { de asistencia, etc. }\end{array}$ \\
\cline { 2 - 2 } & Actividades propuestas y recursos requeridos. \\
\cline { 2 - 2 } & Organización de los estudiantes. \\
\cline { 2 - 2 } & Papel cuando los estudiantes trabajan. \\
\hline $\begin{array}{l}\text { Actuación } \\
\text { de los } \\
\text { estudiantes }\end{array}$ & Actividad para desarrollar el trabajo. \\
\cline { 2 - 2 } & Papel en las actividades. \\
\hline
\end{tabular}

\section{Construcción social del conocimiento}

En las décadas pasadas, el aprendizaje ha pasado de considerarse una actividad individual a verse como una actividad social (Yackel, 2000). También, recientemente, a raíz de la preponderancia del constructivismo, se parte del supuesto de que el individuo construye su conocimiento (Carpenter, Fennema y Franken, 1997; NCTM, 1989, 2000; Sfard, 1996), más que asimilarlo y por consiguiente, este conocimiento no es una copia o imagen de la realidad exterior. De este modo, la conocida pedagogía de la trasmisión, en la que el conocimiento se ve como un paquete que se lleva del profesor al estudiante, es decir el profesor imparte un conocimiento en forma de discurso $^{2}$, el cual es trasladado al estudiante y asimilado por él tal y como se le presenta (Moreno, 1997), está revaluada en la investigación.

La perspectiva filosófica del conocimiento y el aprendizaje denominada constructivismo, atribuye al sujeto que conoce un papel decisivo en la construcción del conocimiento, y supone que éste tiene su origen en la acción mutua

\footnotetext{
2 Se debe tener en cuenta que aquí el término discurso se está usando en el sentido usual del lenguaje común, acertadamente descrito por Ponte, Boavida, Graça y Abrantes (1997): "una intervención larga de un orador, generalmente revestida de formalidad".
} 
del individuo y su medio, físico o social, es decir, en la experiencia del individuo (Waldegg, 1998). El constructivismo moderno puede ser visto como una derivación directa del trabajo de Piaget en epistemología genética, a través de sus teorías de construcción del conocimiento por adaptación cognitiva en términos de acomodación de la experiencia en esquemas de acción (Jaworski, 1994). Pero mientras Piaget ignora las implicaciones sociales y contextuales de las tareas del pensamiento, la corriente constructivista conocida como constructivismo social que se atribuye a Vygotsky, pone mayor énfasis en el individuo como parte de una sociedad (Waldegg, 1998), y asigna un papel determinante al proceso social de discusión y negociación en la construcción de conocimiento por parte del sujeto. Para Vygotsky (1978), "el aprendizaje humano presupone una naturaleza social especial y un proceso por el cual los niños crecen en la vida intelectual de los que los rodean", y ocurre cuando se comparte conocimiento con pares. Ernest (1991) identifica dos características claves del constructivismo social: la construcción activa del conocimiento, conceptos e hipótesis, con base en experiencias y conocimiento previo que guían futuras acciones, y el papel esencial que tienen la experiencia e interacción con el medio físico y social.

Aunque el constructivismo no es una teoría de la enseñanza y aun en su forma social no habla de la enseñanza, según Kilpatrick (1987, citado en Jaworski, 1994) los constructivistas han buscado derivar implicaciones para la práctica; en consecuencia, ciertas prácticas y visiones de la instrucción, presuponen una visión constructivista del conocimiento y el aprendizaje. Por ejemplo, Von Glasersfeld (1987a, citado en Jaworski, 1994) sugiere que se separen los procedimientos educacionales que apuntan a generar comprensión (enseñanza) y los que apuntan a repetir conductas (entrenamiento); que el interés del educador se centre en lo que se puede inferir de lo que pasa en la cabeza del estudiante, más que en las respuestas explícitas; que el profesor se dé cuenta de que no puede transferir el conocimiento al estudiante por comunicación lingüística, sino que el lenguaje puede ser usado como herramienta para guiar la construcción del estudiante; que el profesor se interese en los errores del estudiante, y vea las instancias donde los estudiantes se desvían del camino esperado, como fuentes de luz sobre cómo los estudiantes están organizando sus experiencias del mundo. Waldegg (1998) subraya el papel central que desempeña el profesor en los enfoques constructivistas para ofrecer situaciones interesantes y contextos diferentes, animar y guiar la discusión con el fin de que los estudiantes alcancen las metas cognitivas definidas, aclarar ideas, afirmar conceptos, proporcionar términos y formalizar el conocimiento.

Sin embargo, Sfard $(1996,1998)$ sostiene que es la metáfora de la adquisición la que subyace a estas teorías, pues el aprendizaje se sigue viendo como un acto de ganar conocimiento, es decir, los conceptos se ven como unidades básicas de conocimiento que se pueden acumular, refinar gradualmente y combinarse para formar estructuras cognitivas cada vez más ricas y más complejas; y esto es similar cuando se habla de que las personas construyen significado. 
Esta aproximación al aprendizaje, que parece natural, lo presenta como una actividad de enriquecerse con mercancías o bienes, la mente se ve como un contenedor que debe ser llenado con estas mercancías, y convierte al estudiante en el dueño de estos bienes. Esta autora añade que dado que en cualquier versión del constructivismo que se considere, es central la idea del individuo como constructor y reconstructor de sus concepciones privadas a partir de material externo, permanece el dilema de que los estudiantes sean capaces de construir por ellos mismos conceptos que sean totalmente congruentes con los de otras personas. Además, las teorías basadas en la adquisición casi no dejan espacio para el papel de los intereses genuinos de quienes aprenden, quienes enseñan y quienes deciden qué se debe enseñar; por tanto, si uno espera considerar estos aspectos, la metáfora de la adquisición no es suficiente, ni es la única alternativa para el aprendizaje.

Una nueva visión del aprendizaje como integración a una comunidad en acción más que como un intento de enriquecer una posesión individual, es denominada por Sfard $(1996,1998)$ como la metáfora de la participación. En ésta no hay un punto final claro para el aprendizaje, las actividades matemáticas nunca se consideran separadas del contexto dentro del cual ocurren y este contexto, a su vez, es rico y variado; los términos discurso y comunicación son relevantes y el estudiante se ve como una persona interesada en la participación en una cierta clase de actividades, más que en la acumulación de posesiones. Dicho de otra manera, se concibe el aprender matemáticas como un proceso de convertirse en miembro de una comunidad matemática. Así, para Sfard (1996, 1998, 2000a, 2001), la construcción de conocimiento equivale a ser capaz de participar en un discurso específico. El vocablo "discurso" se entiende como el modo en que los participantes atribuyen significados en situaciones concretas y contextualizadas, el cual supone tanto las ideas y la forma en que éstas se presentan como lo que se relaciona implícitamente con ellas (Ponte, Boavida, Graça y Abrantes, 1997), y abarca cualquier forma de comunicación, bien sea escrita, oral, gestual e incluso mental de una persona consigo misma (Sfard, 2000a, 2000b).

En el trabajo que tiene como foco la cultura de la clase para hacer diseño de instrucción, Cobb y McClain (2001) incentivan normas de participación, como la necesidad de argumentar y las relativas a asuntos matemáticos específicos que ameritan una discusión seria. De acuerdo con estos investigadores, su intención al incluir discusiones de todo el grupo en la estructura de actividades de clase, no es simplemente proveer una ocasión para compartir razonamientos, sino generar discusiones significativas que puedan servir como recurso fundamental para inducir valores, creencias y maneras de aprender en matemáticas. Su interés principal concierne a la calidad de dichas discusiones como eventos sociales en los que participan los estudiantes, y al valor de una discusión sólo cuando los tópicos explícitos de discusión son aspectos matemáticos relevantes que posibilitan avanzar en la agenda de enseñanza.

La construcción de conocimiento enmarcada en las teorías del construc- 
tivismo social, involucra componentes de distinta índole relativos al proceso cognitivo que lleva a cabo el individuo que conoce, a la naturaleza del conocimiento y las características que se le atribuyen a la cognición, a los principios metodológicos que rigen la construcción del saber (Waldegg, 1998), a la validez del conocimiento y al valor que le asigna el sujeto que conoce al conocimiento. Aun así, tanto en estas teorías como en la metafora de la participación, las dinámicas en las que se involucra la persona para aprender, son un componente definitivo. También para Cantoral (2001) la visión sociocultural del conocimiento está relacionada con la base social de los procesos de pensamiento que favorecen la construcción de conocimiento, es decir, con las interacciones sociales de las que tales procesos dependen.

La discusión es, entonces, la actividad vital en este enfoque de construcción social del conocimiento; ésta se concibe como una interacción amplia en torno a un mismo interés o propósito, en la que participa todo el grupo de estudiantes, o gran parte de él, con el profesor y entre ellos mismos, donde hay preguntas que cuestionan y retan a los estudiantes, que exigen explicaciones y razones, se exponen diferentes estrategias y soluciones que se comparan y analizan, los estudiantes expresan sus ideas; las argumentan; comentan las propuestas de sus compañeros; explicitan sus percepciones, dudas y dificultades; exhiben sus reconceptaulizaciones; se acuerdan conclusiones entre todos.

En el Nuevo Proyecto Curricular de la Licenciatura (Departamento de
Matemáticas-UPN, 2002), se espera que en los espacios académicos se propicie construcción social de conocimiento enmarcada en una visión sociocultural del conocimiento y de su didáctica, mediante la simulación en clase de una microsociedad científica donde se construye y reconstruye conocimiento, se combina el trabajo individual con el cooperativo, se moviliza la reflexión, se propicia la expresión libre de ideas y su argumentación.

En esta categoría, se atiende primordialmente a las situaciones que se propician con el fin de favorecer una construcción social del conocimiento. Así se recoge información de las clases acerca del intercambio de ideas y de las discusiones. También se incluye aquí información sobre una mirada sociocultural del conocimiento, conceptualizada, en primer lugar, desde el punto de vista de la manera en que el conocimiento se conoce por los estudiantes, es decir, si se socializa, discute, negocia y acuerda entre todos. En segundo lugar, desde la perspectiva del tipo de tareas que se trabajan si se ven como problemas que logran involucrar activamente al estudiante y que éste los asuma como problemas propios, e implican encontrar un camino donde se desconocía camino alguno, segun Pólya (1957) los caracteriza. En tercer lugar, desde los contextos de la vida cotidiana que se trabajan en estas tareas, si se refieren a situaciones de la vida real relevantes y esenciales, a las matemáticas mismas o a una realidad construida o semirrealidad (Skovsmose, 2000), o a situaciones artificiales o de contexto camuflado (De Lange, 1995). 
Tabla 2. Construcción social del conocimiento.

\begin{tabular}{|c|c|}
\hline \multirow{3}{*}{$\begin{array}{l}\text { Oportunidades } \\
\text { para el intercambio } \\
\text { de ideas }\end{array}$} & Proposición de actividades colectivas. \\
\hline & Trabajo cooperativo. \\
\hline & $\begin{array}{l}\text { Tipo de preguntas: que cuestionan, retan, exigen explicaciones o razones, piden } \\
\text { argumentar las respuestas. }\end{array}$ \\
\hline \multirow{6}{*}{$\begin{array}{l}\text { Desarrollo de } \\
\text { discusiones }\end{array}$} & Participación de los estudiantes. \\
\hline & Interacción entre todo el grupo, gran parte de él, algunos participantes. \\
\hline & $\begin{array}{l}\text { Sujeto que inicia, termina el intercambio, extensión de éste, intervención solicitada } \\
\text { por el profesor o por iniciativa del estudiante. }\end{array}$ \\
\hline & $\begin{array}{l}\text { Presentación, comparación, análisis de diferentes estrategias, soluciones } \\
\text { y respuestas. }\end{array}$ \\
\hline & Expresión de ideas y argumentos por parte de los estudiantes. \\
\hline & Explicitación de dudas y dificultades por parte de los estudiantes. \\
\hline \multirow{3}{*}{$\begin{array}{l}\text { Tratamiento } \\
\text { del conocimiento }\end{array}$} & Negociación y concertación entre todos. \\
\hline & Planteamiento de problemas. \\
\hline & Tareas con contextos. \\
\hline
\end{tabular}

\section{Formación de maestros y ciudadanos}

Los planteamientos acerca de la función que debe desempeñar la educación matemática en la formación para la democracia, proporcionan a dicha educación una dimensión más acorde con las necesidades de la sociedad actual. Autores como Christiansen (1999) y Skovsmose (1999) destacan la necesidad de una educación matemática crítica que permita a los ciudadanos ejercer una competencia democrática, de la cual "el conocer" es una competencia particular. "Una forma básica de aprender los valores democráticos es de hecho la participación en una vida democrática" (Skovsmose, 1999). En consecuencia, los ambientes de formación para los estudiantes deben ser manifestaciones de las formas de organización social deseables. En un sentido similar, Bishop y Clarkson (1988) enfatizan la urgencia de formar valores en los estudiantes de manera consciente a través del trabajo en las clases de matemáticas; para estos autores los valores son cualidades afectivas profundas relacionadas con las creencias: "los valores son creencias en acción", es decir, cuando las creencias se operacionalizan en clase se vuelven valores. Sus análisis revelan que entre los valores que los profesores pueden transmitir, hay valores de la educación general, de la educación matemática y de las matemáticas mismas. Esta distinción se puede equiparar a las diferencias que Yackel y Cobb (1996) encuentran en las prácticas normativas de la actividad de profesores y estudiantes: normas sociales, normas sociomatemáticas y prácticas matemáticas de clase ${ }^{3}$.

\footnotetext{
3 Las normas sociales para Cobb y Yackel (1996) están relacionadas con las creencias acerca del papel del profesor y de los estudiantes, y de la naturaleza de la actividad matemática escolar; por ejemplo, la necesidad de explicar y justificar las soluciones que se presenten durante la clase. Las normas sociomatemáticas aluden a lo que cuenta en clase como una solución matemática diferente, una solución sofisticada, una solución eficiente, y una solución aceptable o válida. Las prácticas matemáticas se refieren a la actividad matemática misma que se da en clase comúnmente y a las concepciones que ellas ponen en juego.
} 
En la actualidad se proponen en el ámbito de la educación matemática, formas de trabajo para favorecer la formación ciudadana, que en muchos casos propenden por trabajar bajo las orientaciones contempladas por los autores mencionados, como el enfoque por proyectos que busca desarrollar una competencia democrática crítica por medio de que los estudiantes conozcan y participen analíticamente en actividades de clase relacionadas con la política, economía o cultura, propias del país. O como la resolución de problemas, las socializaciones y discusiones en el aula, el trabajo colectivo, actividades desde las cuales se intenta fomentar valores específicos al generar normas y reglas que regulen los intercambios, compromisos, acuerdos o consensos y diferencias o contradicciones, y que propicien la vivencia de acciones como escuchar, respetar y tolerar a los demás, tomar decisiones, colaborar con los compañeros, formular y aceptar críticas, comprometerse y asumir el trabajo de manera autónoma y responsable.
Desde este ángulo se generan nuevos requerimientos para la formación de profesores como profesionales de la educación y ciudadanos, teniendo presente que las futuras prácticas de enseñanza de los profesores en formación están permeadas por sus experiencias como estudiantes. En los planteamientos del Nuevo Proyecto Curricular (Departamento de Matemáticas-UPN, 2002) se destaca la importancia de la formación de educadores matemáticos comprometidos y promotores del desarrollo social, moral y ético del país, por medio de alcanzar una actitud crítica, de cooperación, participación, liderazgo, trabajo en equipo y autonomía.

Se recogen entonces en esta categoría las actuaciones de los participantes encaminadas a la formación docente y como ciudadanos de los futuros profesores. Cabe destacar que para la segunda sólo se tuvieron en cuenta las actuaciones relacionadas con el desarrollo de valores de la educación general, según la clasificación de Bishop y Clarkson (1988) o de las normas sociales planteadas por Cobb y Yackel (1996).

Tabla 3. Formación de maestros y ciudadanos.

\begin{tabular}{|l|l|}
\hline $\begin{array}{l}\text { Actuaciones para } \\
\text { la formación } \\
\text { ciudadana }\end{array}$ & $\begin{array}{l}\text { Intervenciones y actividades dirigidas a promover } \\
\text { la ética, los valores y principios de vida. }\end{array}$ \\
\cline { 2 - 2 } \begin{tabular}{l} 
Intervenciones y actividades indirectas que podrían servir de ejemplo. \\
\hline \multirow{2}{*}{$\begin{array}{l}\text { la formaciones para } \\
\text { como maestros }\end{array}$}
\end{tabular} & $\begin{array}{l}\text { Intervenciones y actividades encaminadas a promover o destacar comportamientos } \\
\text { deseados para los docentes de matemáticas. }\end{array}$ \\
\cline { 2 - 2 } & Intervenciones y actividades indirectas que podrían servir de ejemplo. \\
\hline
\end{tabular}

\section{Evaluación para la promoción de los estudiantes}

Las tendencias investigativas actuales involucran una conceptualización de la evaluación del aprendizaje matemático que según Romberg (1988, citado en
Rico, 1990), ha evolucionado desde una concentración sobre la medida de los logros para hacer juicios de los estudiantes, a un interés en proporcionar 
información para apoyar políticas y toma de decisiones. Rico (1990) añade que el punto de vista actual se centra en que para evaluar hay que comprender el proceso de aprendizaje, es decir, hacer un juicio razonado sobre el trabajo, lo cual difiere del enfoque convencional, en el que se trata de establecer un éxito o un fracaso. La evaluación se considera un elemento central del currículo, en el que se plantean cuestiones más amplias que establecer el progreso o la promoción de los estudiantes a través de los distintos grados escolares, por ejemplo, como medio de diagnóstico, de superación de situaciones anómalas (Rico, 1995) y a su vez como vía de instrucción que posibilita el aprendizaje. De Lange (1995) insiste en que la primera meta de la evaluación debe ser mejorar la enseñanza y el aprendizaje, y agrega que la evaluación debe operacionalizar todos los objetivos de la educación.

Realizar varias actividades de distinto tipo con el objeto de recolectar gran y diversa cantidad de información sobre el trabajo de los estudiantes, es indispensable para contribuir a la máxima objetividad en la evaluación, para que los estudiantes puedan demostrar lo que saben o no, para informar a los interesados sobre los avances del estudiante, para que el mismo estudiante pueda identificar sus dificultades y trabajar en ellas, y para que el profesor y las instituciones puedan tomar acciones encaminadas a mejorar la enseñanza (NCTM, 1989, 1991, 2000; Kilpatrick, 1995). De este modo se deberían emplear diferentes tareas para evaluar, como proyectos, preguntas abiertas, resolución de problemas, cuestionarios, entrevistas, exposiciones, etc., y utilizar instrumentos como portafolios, autoevaluación, coevaluación. Por otra parte, proponer tareas que permitan abordar un mismo tema desde distintos ángulos es deseable no sólo para ayudar a su comprensión sino además a su evaluación (Rico, 1990).

Este estudio se enfocó en conocer las estrategias de evaluación implementadas en los espacios académicos, mediante las cuales los estudiantes acceden a cursos subsiguientes, y en determinar su coherencia con la innovación de enseñanza y aprendizaje puesta en práctica.

Tabla 4. Evaluación para la promoción de los estudiantes.

\begin{tabular}{|l|l|}
\hline \multirow{4}{*}{$\begin{array}{l}\text { Estrategias } \\
\text { de } \\
\text { evaluación }\end{array}$} & Forma y contenido de las tareas y actividades de evaluación. \\
\cline { 2 - 2 } & $\begin{array}{l}\text { Instrumentos como portafolios, autoevaluación, coevaluación. } \\
\text { Logros y criterios para determinar el avance de los estudiantes. }\end{array}$ \\
\cline { 2 - 2 } & $\begin{array}{l}\text { Aspectos que se tienen en cuenta: de orden conceptual, procedimental, informativo, } \\
\text { actitudinal, etc. }\end{array}$ \\
\cline { 2 - 2 } & Seguimiento que se hace. \\
\cline { 2 - 2 } & Forma de establecer la nota final del curso. \\
\hline
\end{tabular}




\section{Desarrollo del contenido}

La nueva dimensión del conocimiento profesional del profesor de matemáticas, conlleva a la reconceptualización del conocimiento acerca del contenido matemático y su relación con el conocimiento de la didáctica de las matemáticas, al introducir aspectos relativos al uso que el profesor hace de los temas en las situaciones de enseñanza. Según Llinares (1994), se reconoce que el conocimiento del profesor puede adoptar diferentes formas al ser explicitado. El contenido que se aborda en clase y la manera en que se trata son reflejo de este conocimiento, pero también de las visiones del profesor acerca de lo que son la enseñanza y el aprendizaje. Romberg (1991) sostiene que aunque la elección de qué matemáticas enseñar a los estudiantes es relevante, dado que cambia con cada cultura y ha evolucionado con el desarrollo de las sociedades tecnificadas, lo verdaderamente importante es lo que significa "saber" matemáticas para el profesor, y por tanto lo que hace en clase para que los estudiantes "sepan". Para algunos, saber matemáticas es conocer principios, definiciones, enunciados y su aplicación; para otros, es "hacer matemáticas" en el sentido propuesto en los estándares curriculares del NCTM (1989, 2000). También, en el Nuevo Proyecto Curricu- lar se ha propuesto la redefinición de los contenidos de los espacios académicos, y en algunos el diseño curricular y, por tanto, el contenido estudiado ya es producto de investigaciones realizadas por el Departamento de Matemáticas sobre la enseñanza y el aprendizaje de tópicos matemáticos particulares.

A través de las actividades que se realizan y las tareas que se proponen en clase, es posible identificar el contenido estudiado y la posible intencionalidad respecto a lo que debe enfatizarse y se quiere que los estudiantes aprendan. Ponte, Boavida, Graça y Abrantes (1997) afirman que las tareas propuestas por el profesor y las actividades realizadas por los estudiantes son un elemento decisivo en la dinámica de la clase de matemáticas y en los procesos de enseñanza y aprendizaje.

Esta categoría apunta a determinar tanto el contenido abordado como el camino recorrido en clase para ello. En particular, el contenido se considera construido por hechos, técnicas, destrezas, estrategias, conceptos y estructuras conceptuales en el sentido sugerido por el informe Cockroft (1985) y por Rico (1990, 1995); es decir, esta organización abarca tanto la información matemática, sus relaciones, sus representaciones, como los procesos que se llevan a cabo para usarla.

Tabla 5. Desarrollo del contenido.

\begin{tabular}{|c|c|}
\hline \multirow{5}{*}{ Proceso } & Secuencia y organización temática. \\
\hline & $\begin{array}{l}\text { Nociones, conceptos, definiciones y notaciones tratadas, procedimientos ilustrados y empleados, } \\
\text { representaciones utilizadas, traducción entre ellas. }\end{array}$ \\
\hline & Sujeto que interviene o desarrolla la actividad. \\
\hline & $\begin{array}{l}\text { Tareas específicas de la asignatura, tipo de actividad matemática implicada (resolver problemas, } \\
\text { seguir procedimientos, hacer matemáticas, generalizar, conjeturar, razonar matemáticamente, } \\
\text { comunicar ideas, hacer conexiones, consultar información); contextos propuestos para las tareas. }\end{array}$ \\
\hline & Explicaciones, argumentos, justificaciones. \\
\hline
\end{tabular}




\section{Resultados}

Los resultados encontrados que se presentan a continuación, atendiendo a las categorías contempladas para el exámen de las rutas pedagógicas, ilustran la complejidad de los aspectos seleccionados y la vigencia de los mismos para la comunidad académica de educadores matemáticos; también permiten cuestionar y confrontar las rutas que realmente se siguen como operacionalización de los planteamientos delineados.

\section{Metodología}

La proliferación de reformas educativas que se han dado a nivel internacional y nacional, en épocas recientes, no son ajenas a los educadores, y es claro que la información que obtienen de estas propuestas innovadoras los ha llevado a introducir nuevos términos en su vocabulario, como cuando dicen: "Con la metodología propuesta se espera que... el estudiante participe activamente y con compromiso en grupos de trabajo", "Se convierta en un miembro propositivo de grupos de trabajo”, “Toma conciencia que el papel fundamental del docente es ser facilitador del aprendizaje", "Propiciar la participación en una serie de actividades de investigación”. Quizás también ha originado la implementación de algunos cambios en sus clases, reflejados por ejemplo, en la ausencia de clases magistrales en los cursos observados, en el sentido de que el profesor actúe como orador durante toda la sesión de clase y los estudiantes permanezcan callados escuchando y tomando notas; si bien en las clases hay exposiciones por parte del profesor, en general, a medida que los profesores van exponiendo, intercalan preguntas para los estudiantes, como se ve en el siguiente fragmento de clase.

Profesor. Cuando nos aproximamos a 1 por derecha podemos tomar valores próximos a 1 evaluándolos en $f(x)=\left(-x^{2}+5 x\right) / 2$ porque la función está definida así; de igual forma para valores menores o iguales a 1 los evaluamos con la función $f(x)=$ $2 x$... Con la tabla vemos que cuando nos aproximamos por la derecha de $x=1$ los valores de $f(x)$ se acercan a 2 , y de igual forma cuando nos acercamos por izquierda los valores se aproximan a 2, de aquí concluimos que cuando $x$ tiende o se acerca a 1 , $f(x)$ tiende a $2 \ldots$ iTienen alguna duda hasta aquí?

Esas exposiciones y de manera global la metodología puesta en práctica en los espacios académicos observados, se perciben permeadas en cierta medida por planteamientos que se refieren a crear oportunidades para incluir al estudiante de manera más activa en clase. Así, además de responder las preguntas del profesor cuando expone, pueden distinguirse en las clases de estos espacios académicos, otras acciones metodológicas que involucran a los estudiantes, y que varían en cuanto a la frecuencia y a la forma dependiendo del profesor: desarrollar las tareas propuestas por el profesor, individualmente o en pequeños grupos, trabajar en el tablero para reelaborar tareas ya realizadas o a veces para desarrollar nuevas tareas, exponer trabajos que fueron asignados anteriormente, contestar las preguntas que el profesor formula en torno al trabajo que los mismos estudiantes llevan a cabo.

También se destacan otros aspectos metodológicos valiosos: la forma que 
uno de los profesores emplea para dirigirse a los estudiantes que logra establecer una relación de confianza y camaradería con ellos, la cual vemos que aporta a una participación nutrida y más abierta y desinhibida, que se traduce en expresiones del tipo: "Yo de probabilidad no sé mucho", "Yo también estoy un poco confundido y me toca ponerme a leer sobre eso"; las variadas opciones de trabajo que otro profesor genera con tareas diversas que se complementan y contribuyen, quizás, a una mejor comprensión del contenido abordado, como plasmar un mismo programa de computador en un algoritmo, en instrucciones de un lenguaje, en un diagrama de flujo; la oportunidad que un profesor ofrece a los estudiantes para que socialicen las distintas conjeturas o soluciones que han encontrado a un problema, y sean escuchadas por todos, ilustrada en las siguientes indicaciones: "En cinco minutos alguien habrá terminado el primero, algún grupo lo expone y continuamos", "A ver, por favor, el primero"; las preguntas cuestionadoras y abiertas planteadas por unos de los profesores para los estudiantes, que en unos pocos casos son motivo de debate, como: “¿Qué es una ecuación?", y a la respuesta: "Una igualdad”, el profesor escribe: “ $x=3$ ” y dice: "Ustedes dijeron que era una igualdad"; la posibilidad que dos de los profesores proveen a los estudiantes para que avancen a su ritmo en el desarrollo de trabajos, y cuando consideren que están listos, los expongan.

Aun así, y a pesar de que la metodología observada varía dependiendo del área de formación de los cursos observados y por supuesto del profesor, en general en los espacios de matemáticas considerados, la metodología no presenta mayores diferencias con la que ha sido usual en la enseñanza de unos años atrás, y que en algunos estamentos del país se ha llamado convencional, debido a que pese a haber introducido algunas transformaciones con respecto a la enseñanza conocida como tradicional, no son cambios sustanciales que se reflejen por ejemplo, en la forma de abordar los temas matemáticos, y se ha quedado estancada al comienzo del camino (Andrade, Perry, Guacaneme y Fernández, 2003b). Por tanto, no podría afirmarse que esta metodología esté ilustrando la teoría acerca de corrientes didácticas o modelos pedagógicos, estudiada en los espacios académicos del ambiente de pedagogía de la Licenciatura, ni se vea como ejemplo especial para la futura labor de los estudiantes; tampoco se podría aseverar que la metodología refleje en toda su dimensión las indicaciones que para el Nuevo Proyecto Curricular se han especificado respecto a que se posibilite que los estudiantes desarrollen conocimiento didáctico, reflexionen sobre la enseñanza, se inicien en la actividad profesional y se acerquen a la investigación.

Por otra parte, vale la pena destacar que el malestar de los estudiantes cuando se implementan en clase metodologías que involucran cambios de fondo respecto a las usuales, junto con los resultados poco satisfactorios de los estudiantes, llevan a los profesores a acomodarlas a formas de trabajo familiares para los estudiantes, las cuales producen confianza en relación con lo que se espera tanto de ellos como del profesor mismo. Algunos estudiantes manifiestan en la entrevista: "Los prime- 
ros talleres no me gustaron... Nosotros no recordábamos algunos temas y él [el profesor] ya los asumía, entonces no nos dio como una guía de las cosas que podríamos necesitar para resolver talleres sino, ahí les dejo para que resuelvan, y teníamos que entregar el taller; entones teníamos como muchas dudas... Después, como a mitad de semestre hacia arriba, él ya empezó con otra tónica: nos explicaba algunas cosas y después de eso ponía ejercicios como para ayudar a crear la discusión entre nosotros, a mirar cómo lo solucionaríamos, a decir quién pasa al tablero, cosas así, ya como que uno se motiva más", "Lo que yo le digo: no tenía ni idea del tema; y decía: ¿cómo voy a desarrollar un taller de algo que yo no sé? Entonces, hacía falta que él nos explicara algunas cosas primero para hacer los ejercicios".

Se cree, que a pesar de que estos talleres incorporan situaciones para el aprendizaje en las que el estudiante debe explorar estrategias y formas de razonamiento para acercarse al tema, el rechazo a los cambios se origina precisamente en que éstos son puntuales, no se implementan de forma completa y no se consideran suficientemente las eventuales dificultades ni posibles desvíos de la trayectoria planeada. Por ejemplo, desarrollar talleres en grupos de estudiantes requiere también de discusiones dirigidas en cada grupo, de socializaciones a nivel de todo el curso, de concertación de resultados entre todos, de preguntas y tareas adicionales y complementarias; asumir una responsabilidad e independencia frente al propio aprendizaje por medio de un trabajo autónomo, necesita de directrices y vivencias iniciales que permitan definir métodos y disciplina de trabajo, fijar metas y, en general, desenvolverse en este sentido.

\section{Construcción social del conocimiento}

Las conclusiones en esta categoría se refieren a construir conocimiento socialmente, es decir, a través de una interacción con otras personas, en la que los participantes son interlocutores unos de otros y que comparten un mismo interés. De ningún modo, estamos diciendo que no puede haber construcción de conocimiento o aprendizaje mediante otros mecanismos, y por supuesto estamos lejos de negar que en los espacios académicos observados, los estudiantes puedan haber construido conocimiento.

De acuerdo con las intenciones manifestadas por los profesores con respecto a la construcción social de conocimiento, como: "El conocimiento sea fruto de una construcción grupal o social", "Búsqueda por el desarrollo social del estudiante y de participación activa en el proceso de enseñanza aprendizaje", "Implementar en el aula procesos de discusión, socialización y validación", es claro que todos tienen presente la posible relevancia de este aspecto en sus clases, pero asociada principalmente con la participación de los estudiantes en clase, la cual se espera en las actividades propuestas; se solicita que el estudiante realice el trabajo asignado, conteste oralmente algunas preguntas, presente dudas y exponga su trabajo en el tablero. No obstante, las alusiones explícitas que piden la participación activa de los estudiantes, no expresan qué tipo de participación se considera como activa; no se dan pautas indicadoras del contenido o de la forma de las intervenciones esperadas; por ejemplo, que las respuestas sean com- 
pletas o argumentadas; no hay indicios de un seguimiento sistemático de la participación y aunque quizás el registro que se hace sobre el trabajo realizado y entregado por los estudiantes, podría ser considerado por algunos profesores como termómetro de la participación, no es claro de qué manera se pudo hacer; tampoco hay señales de que la participación se haya tenido en cuenta de alguna forma para la nota final de los estudiantes.

Con algunas excepciones, el trabajo en clase no se acompaña de acciones deliberadas en el aula para instigar a los estudiantes a compartir, socializar, argumentar y consensuar sus ideas, de manera que la participación de los estudiantes sea más sustancial y se vincule más esencialmente a la comprensión del contenido tratado. Por ejemplo, se formulan preguntas puntuales las cuales generan respuestas del mismo tipo que no incitan a ser discutidas como: "¿Cuál es la derivada de $f(x)=x^{2}-5 x+6 x$ ?". O cuando las preguntas son de otro estilo y hay respuestas, éstas en general no se exploran ni ahondan; es el caso de la pregunta: “¿Qué es lo primero que toca hacer?", un estudiante responde: "Multiplicar por el conjugado" y se finaliza la interacción. Si no hay respuesta, el profesor termina por suministrarla, como la situación donde los estudiantes permanecen callados después de que se pregunta por características de una gráfica cartesiana, y entonces el profesor dice: "También podemos mirar en qué intervalos la función es creciente o decreciente, en dónde es cóncava hacia arriba y cuándo hacia abajo, etc.”.

Se plantean preguntas que pueden significar un reto o cuestionar a los es- tudiantes y normalmente se dejan en el aire para que ellos las investiguen por su cuenta, pero luego no se retoman, como: “¿Quién demostró el teorema de Fermat?”, “¿Saben cuándo apareció el cero?”, “¿Quién de los matemáticos es el más prolífero en la historia de las matemáticas?”.

Se hacen preguntas o se formulan tareas que fomentan la participación de muchos estudiantes, pero dado que no es fácil encausar las múltiples respuestas obtenidas hacia el tópico del conocimiento deseado, se considera generalmente la respuesta adecuada; así lo señala un estudiante:

En el transcurso de la clase, él siempre nos incita primero a ver el ejercicio, a analizarlo... Para que nosotros lo solucionemos de alguna forma y espera a ver qué clases distintas de solución se le dan al mismo problema, entonces empieza a coger por ahí, ya después nos dice lo que él trae listo y la forma como él lo hace.

No es común la concertación o acuerdo de ideas y resultados entre todos, ni el establecimiento de formas claras de regular dicha concertación. El profesor usualmente es quien termina por validar las ideas y concluir, o no se concluye; por ejemplo en clase un profesor pregunta: "Algebraicamente, ¿qué es la derivada?... Y, ¿geométricamente?", y ante la respuesta: "Una tangente a la curva", señala: "No es lo mismo la tangente en la geometría euclideana que en la geometría analítica. En la euclideana se habla de tangente a toda la curva, y en la analítica, de tangente en intervalos pequeños". En el siguiente fragmento de clase, el profesor es quien sintetiza. 
Estudiante $_{1}:$ ¿Cuál es el que multiplicado por el mismo da el mismo?

Estudiante $_{2}$ : Las diagonales en un sentido están formadas por el mismo número.

Estudiante $_{3}$ : Relación entre las dos: multiplicar por 11 es el mismo resultado pero inverso que al sumarlos con cero...

Profesor: Yo descubrí que para la multiplicación hicieron trampa...

Estudiante $_{4}$ : En la suma hay secuencia.

Estudiante 2 : Los números en diagonal son iguales en un solo sentido.

Estudiante $_{5}$ : Todo número sumado con cero nos da el mismo número.

Estudiante $_{6}$ : Al llegar a 11 inicia un nuevo ciclo. Horizontal y verticalmente son la misma secuencia.

Profesor: La suma da siempre un número entre 0 y 11 entonces es cerrada.

Casi siempre, los intercambios se dan entre el profesor y uno o dos estudiantes, y raramente participan más estudiantes; estos intercambios se parecen más a conversaciones guiadas por el profesor, donde el profesor pregunta y los estudiantes contestan, el profesor vuelve a preguntar y es escasa la interacción entre los mismos estudiantes. Esto se ilustra en el siguiente fragmento de clase.

Profesor: ¿Qué pasa con $f_{2}$ respecto a

$f_{1}$ ? (Se refiere a las funciones $f(x)=|x|$ y $f(x)=|x-1|)$.

Estudiante: Se corre una unidad a la derecha.

Profesor: Pasa y grafícala.
Profesor: Efectivamente se desplaza una unidad a la derecha u horizontalmente. ¿Quién pasa y grafica $f_{3}$ y $f_{4}$ ? (Se refiere a las funciones $f(x)=|x|-1$ y $f(x)=|x-1|-1$ ) (otro estudiante pasa y hace las gráficas).

Profesor. ¿Están todos de acuerdo?

En dos de los espacios académicos se registran unas pocas discusiones cercanas a la conceptualización planteada aquí para el término "discusión”, donde pudo haber construcción social de conocimiento, en especial para los estudiantes que participaron en ellas; sin embargo, tales discusiones fueron esporádicas y no fueron la regla común para los cursos; además, podrían enriquecerse con más preguntas y argumentos, con una indagación más amplia de las respuestas obtenidas, y con una mayor concertación de resultados entre todos. En el fragmento de clase que se presenta a continuación se muestra uno de estos intercambios.

Profesor: $5 \mathrm{x}+1+5 \mathrm{x}=750$

Estudiante $_{1}$ : (Escribe en el tablero)

$$
\begin{aligned}
& 5 x+1+5 x=54+53 \\
& x+1+x=4+3 \\
& 2 x+1=7 \\
& 2 x=6 \\
& x=3
\end{aligned}
$$

Profesor: Bueno.

Estudiante $_{2}$ : Profe, ¿se puede hacer así?

Profesor: Espere, de poderse hacer se puede. Ya está hecho. El logaritmo de la suma no es la suma de los logaritmos (escribe en el tablero) $\log _{a}(\mathrm{M}+$ $\mathrm{N}) \neq \log _{\mathrm{a}} \mathrm{M}+\log _{\mathrm{a}} \mathrm{N}$.

Estudiante $_{2}: \log (\mathrm{MN})$ y iya! Sale. 
Profesor. Eso ya se había escrito la clase pasada.

Estudiante $_{2}$ : (Escribe en el tablero)

$$
\begin{aligned}
& \log _{25}(5 \cdot 5)^{\mathrm{x}+1+\mathrm{x}} \\
& =\log _{25}(5 \cdot 5)^{7} \\
& \mathrm{x}+1+\mathrm{x}=7 \\
& 2 \mathrm{x}=6 \\
& \mathrm{x}=3
\end{aligned}
$$

Profesor: ¿Qué dicen de eso? Eso sí se puede hacer, si aquí dijese $\log _{25}$. La propiedad dice:

$\log _{\mathrm{a}}(\mathrm{M} N)=\log _{\mathrm{a}} \mathrm{M}+\log _{\mathrm{a}} \mathrm{N}$, pero aquí no tengo eso. ¿Cómo es?

Estudiante ${ }_{3}: \log _{5} 5^{4} \ldots$ (escribe en el tablero)

$$
\begin{aligned}
& \log _{5} 5^{4}+\log _{5} 5^{3} \\
& 4 \cdot 1+3 \cdot 1 \\
& 4+3 \\
& 7
\end{aligned}
$$

Estudiante $_{4}$ : La única forma para que dé así...

Profesor: Que sí se puede hacer: sacar el logaritmo a ambos lados, es decir

$\log \left(5^{(x+1)}+5^{x}\right)=\log \left(5^{4}+5^{3}\right)$ pero no puedo decir nada del logaritmo de una suma, ¿Cómo es que hacen que $5^{4}+5^{3}=\log _{5} 5^{4}+\log _{5} 5^{3} ?$

Profesor: ¿Quién lo hizo ya?

Estudiante $_{1}$ : Nadie, porque yo lo hice así.

Estudiante $_{6}: \quad$ (Escribe en el tablero)

$$
\begin{aligned}
& 5^{(x+1)}+5^{x}=5^{4}+5^{3} \\
& 5^{(x+1)}+5^{x}=5^{3+1}+5^{3}
\end{aligned}
$$

Profesor: Bases iguales, exponentes iguales, ajá; pero se dice es: "potencias iguales", no suma de potencias.
Yo decía que tanto impulso no podía ser cierto.

Estudiante $_{7}$ : (Escribe en el tablero)

$$
\begin{aligned}
& \log _{5} 5^{x+1}+\log _{5} 5 \\
& x=\log _{5}\left(5^{4} \cdot 5^{3}\right)
\end{aligned}
$$

Profesor. Insisten en hacer la suma de los logaritmos. Sugerencia: inicialmente no se hace usando logaritmos.

Estudiante $_{2}$ : ¡Que no! Está diciendo que el logaritmo de una suma es la suma de los logaritmos.

Profesor: Los tipos son unos ases para cuadrar la respuesta.

Estudiante $_{3}$ ¡YYa profe! (Escribe en el tablero)

$$
\begin{aligned}
& 5 x \cdot 5+5 x=6 \cdot 125 \\
& 5 x(5+1)=6 \cdot 125 \\
& 5 x(6)=6 \cdot 125 \\
& 5 x=125 \\
& 5 x=5^{3} \\
& x=3
\end{aligned}
$$

Profesor: Sí señor.

Aunque en uno de los cursos, al comienzo se proponen tareas que involucran contextos relacionados con situaciones de la vida diaria, más tarde esto ya no es lo habitual; en los demás espacios académicos tampoco se registran tareas que impliquen un trabajo con datos de la vida real familiares a los estudiantes. Por todo lo expuesto, es difícil hablar de que en los cursos observados se den discusiones y se fomente un enfoque social para la construcción de conocimiento. Uno de los profesores al final del semestre comenta sobre un logro no explicitado que no alcanzaron sus estudiantes: "Participar y discutir 
sobre clase de matemáticas... Porque ese tendría más de uno que no lo alcanzó, porque no discutieron... Porque algunos solamente asisten".

Un factor que posiblemente influye en que las oportunidades de interacción y socialización en clase sean solamente las que son y se den en la forma que se presentan, podría estar relacionado con las distintas concepciones a las que cada profesor ha llegado con respecto al significado de la expresión "construcción social del conocimiento", a cuya claridad tampoco ayuda la caracterización poco detallada que el documento de condiciones del Nuevo Proyecto Curricular presenta. Otro factor puede estar ligado al papel preferiblemente autónomo que unos de los profesores le adjudican al estudiante en el aprendizaje, que aunque deseable desde todo punto de vista por estar en sintonía con las exigencias de programas universitarios para niveles académicos altos, los empuja a esperar que las discusiones surjan del trabajo que los estudiantes han realizado de manera independiente, con el riesgo de que si esto no sucede o se presentan circunstancias de falta de tiempo o de otra índole, no hay discusión o ni siquiera se tratan los tópicos. Además es factible que también incida la falta de experiencia de los profesores en el uso y manejo de discusiones, por lo cual prevalezca una sensación de temor ante esta actividad y de inutilidad de ella, fundamentada en algunas vivencias previas poco exitosas; como profesores todos hemos vivido las dificultades de comenzar, guiar y coordinar negociaciones matemáticas y discusiones productivas en clase, pues como lo señalan Cobb y Yackel (1996), éstas son actividades altamente comple- jas para las que se necesita haber ganado experiencia, pero si no se empiezan a poner en práctica, dificilmente se obtendrá esa experiencia. Así mismo, factores más externos a los profesores como la obligación de estudiar en el curso unos contenidos definidos y ejecutar ciertas tareas en un tiempo predeterminado, normalmente afectan el trabajo que se lleva a cabo en el aula, y es sabido que para discutir los conceptos que se estudian hay que disponer de más tiempo que cuando simplemente se presenta la definición del concepto, se ilustra la forma de desarrollar ejercicios que lo emplean y luego se proponen ejercicios para realizar.

\section{Formación de maestros y ciudadanos}

Algunas actuaciones de los profesores encargados de los espacios académicos observados pueden ser tomadas por los estudiantes como ejemplos a seguir y, de esta manera, incidir en su formación como ciudadanos y futuros docentes.

En primer lugar se percibe que el acontecer de las clases discurre de a-cuerdo con unas normas de comportamiento social y que el proceder de los profesores revela valores de un ser humano, tales como agradecer al estudiante el trabajo realizado en el tablero con frases como "Ok, muchas gracias", "Bien, ya acabaste, gracias", tratarlo de igual a igual en forma respetuosa y considerada, darle la oportunidad de expresar sus opiniones y escucharlas como cuando un profesor anota: "La idea de este curso también es escucharnos", mostrar interés por asuntos relacionados con la política, la economía y la cultura de las sociedades, por ejemplo al hacer una referencia histórico-política 
en clase sobre El Salvador, e inclusive colaborarle más allá de lo exigido. Presumiblemente esto contribuye a que los estudiantes actúen de forma similar en clase, como efectivamente lo hacen en estos cursos.

También sobresalen positivamente algunos actos de los profesores como docentes, que además fueron reconocidos por los estudiantes en las entrevistas, como formular preguntas que cuestionan el conocimiento de los estudiantes, quedarse callado cuando impera el desorden en el aula, proponer diversidad de tareas y actividades para los estudiantes acerca de un mismo contenido, hacer un seguimiento casi personal al desempeño de los estudiantes, abrir espacios para que se oigan las soluciones individuales.

Estamos convencidos de que aun cuando el ejemplo del actuar del docente puede aportar a la formación, en particular no parece haber sido especialmente eficaz para promover valores en el pasado, ni en la actualidad se percibe como suficiente para moldear la formación como ciudadanos críticos y comprometidos o como maestros innovadores, de estudiantes universitarios que ya traen unas bases al respecto.

En los espacios académicos observados, los profesores hacen declaraciones, acotaciones y comentarios de distinta especie que versan sobre el deber ser de un profesor de matemáticas, como: “...O aprendemos a vencer el miedo [a hablar] o nos morimos de hambre", "El cálculo es lo que nos va a dar de comer. Porque es de lo que más nos llaman para que expliquemos, por eso es bueno que lo practiquemos", "A la hora de ir a enseñar tenemos que desglosar muy bien lo que estemos haciendo y lo que vamos a dictar para no dejar dudas en los estudiantes", "Mire, lo más doloroso que hay es que en Colombia se producen muchas cosas, pero pocas las escriben, y entonces cuando se muere ese personaje, se murió ese conocimiento, porque nadie contó y de pronto ese era el modelo mejor en esa zona, lo mejor del país, porque a ese profesor siempre se le entendió como enseñaba eso, pero él nunca lo escribió, entonces eso murió allá”. Igualmente, los profesores producen anotaciones, invitaciones y reconvenciones acerca del deber ser de un estudiante y de un ciudadano, para respetar normas de convivencia social, para asistir puntualmente a clase, para cumplir con los deberes y compromisos pactados, sobre acontecimientos de la vida pública, de orden político, social, cultural y económico, sobre anécdotas personales y del acontecer profesional, etc., quizás movidos por un convencimiento del posible aporte de ellas en la formación de los estudiantes; ejemplos de esto son los siguientes señalamientos: "Leer en matemáticas no es como leer en literatura, por ejemplo, me preguntaban de dónde salió el tres, yo no sé; hay que tomar un lápiz e ir haciendo", "El mundo cambia y nosotros estamos inmersos, hay que sacar la cabeza... Estudiar, estudiar, leer y leer para entender el mundo".

La cantidad, la fuerza y la vehemencia de estas intervenciones varían según el profesor y claramente en uno de ellos se evidencia una alta propensión a este tipo de arengas en clase. Es claro que de manera natural tales intervenciones envuelven valores, seguramente no pasan desapercibidas para los estudiantes 
y tienen alguna ingerencia en ellos, tal como lo expresa un estudiante en la entrevista:

Yo aprendí que, pues la cuestión no está sólo en centrarse en una sola cosa, pues a mí lo que me enseñó es que no sólo existe la matemática, ¿si?, sino que hay que mirar más allá y hay que saber, en el mundo en que uno se desenvuelve, cómo funciona, qué es lo que está pasando en la realidad... él [el profesor] ha sembrado en mí es eso: como que mire, mire lo que pasa a su alrededor, que lo que vive aquí no es lo único que está sucediendo.

En un caso específico se percibe que entre los estudiantes hay una especial conciencia y aceptación de lo que el profesor dice, al parecer que debido a la relación de cercanía y confianza que logra instaurar con los estudiantes, y así lo señala un estudiante:

El profesor siempre trató de hacernos conscientes de muchas cosas, entre otras, el ejercicio de ser profesor... Entonces él nos hablaba de sus experiencias y siempre era muy reiterativo en decir: "Tengan cuidado con esto, miren, tengan cuidado de esto", pero, infortunadamente, pareciera que solamente, cuando le pasan a uno, cuando la experiencia es propia, es cuando la asume.

A veces dichas anotaciones dispersan la atención de los estudiantes u ocupan gran parte del tiempo destinado al tratamiento de contenido propio del curso; un estudiante comenta al respecto: "De vez en cuando se presentaba el chiste en la clase, entonces a veces eso contribuía a que ciertas personas se dispersaban, que uno se dispersaba y empezaba a hablar con otro". Consideramos que se requiere mirar más críticamente lo que como profesores expresamos a nuestros estudiantes, para inculcar las afirmaciones y recomendaciones que se hacen en clase, de propósitos concretos encaminados a la formación de los estudiantes. De igual modo, se deben examinar las menciones de los profesores que puntualizan cómo debe actuar un profesor, las cuales probablemente concuerdan con sus visiones acerca de lo que debe ser la enseñanza, pero no son generalizables o válidas siempre.

Hay además en los espacios académicos, exhortaciones de los profesores para propender por la responsabilidad, independencia y autonomía de los estudiantes frente al trabajo y su aprendizaje, como: "Ustedes pueden solucionarlo... Ya tienen herramientas", "Ustedes por sí solos deben saber qué errores están cometiendo", que van de la mano con la adjudicación de tareas de consulta, lectura y análisis para las que se suministran básicamente instrucciones generales; también algunos planteamientos de los programas de curso, como "promover la independencia del estudiante y la responsabilidad que se debe tener en el propio trabajo" apuntan a esto. Los estudiantes reconocen estas intenciones al decir: "Por el mismo tipo de asignatura le permite como darle mayor responsabilidad al estudiante, pues es un proyecto a desarrollar durante todo el semestre, y la dinámica misma la va colocando el estudiante; es decir, en la medida en que él avance, el profesor avanza con él", "Lo que el profesor quería hacer era motivarnos a que nosotros mismos estudiemos, que uno no siempre dependa de lo que él siempre le enseña a uno, sino que uno también se ponga a estudiar". Sin embargo, fuera de los señalamientos insistentes del profesor con respecto 
al trabajo que los estudiantes deben realizar, hay muy pocas acciones que puedan apoyar el conseguir esta actitud del estudiante.

En uno de los espacios académicos se proponen unas actividades con la intención de que los estudiantes comenten críticamente el trabajo de sus compañeros, las cuales podrían contribuir a desarrollar la tolerancia y el respeto. Con motivo del cierre y reapertura de la Universidad Pedagógica Nacional durante el semestre en cuestión, los profesores implementaron actividades de reflexión, en las que especialmente en uno de los espacios académicos puede verse una intención del profesor por ejemplificar cómo abordar un conflicto por medio de una reflexión colectiva. No hay información acerca del efecto que esta situación haya podido causar en los estudiantes; en otros de los cursos se registran quejas de los estudiantes sobre la repetición de tal reflexión en todas las asignaturas que cursan.

En definitiva, las situaciones que se dan en las clases observadas para promover una formación ciudadana a través del desarrollo de valores, tal y como la hemos concebido, se reducen al ejemplo del profesor, a las acotaciones de los profesores que en uno de los cursos son reiteradas, a unas actividades puntuales de coevaluación y a la exhortación a que los estudiantes trabajen por sí mismos. Consideramos que adicionalmente se requiere poner en juego acciones que, conjuntamente con las palabras, alienten a los estudiantes, por ejemplo, a actuar de acuerdo con unas normas mínimas para vivir en sociedad, a entrenarse en el camino del estudio autónomo y responsable.
Cabe reconocer las dificultades inherentes a implementar una formación ciudadana, ya que ésta se refiere a un espectro amplio de aspectos integrados entre sí, y ponerla en práctica exige delimitar propósitos y enfocarse en algunos aspectos concretos, de modo que sea posible también aterrizar los lineamientos que al respecto se exponen en el documento de condiciones del Nuevo Proyecto Curricular. Las varias tendencias, ya mencionadas, que se encuentran en la comunidad de educación matemática y que sugieren acciones para la formación ciudadana, obligan tanto a identificar aspectos particulares que sean el centro de sus intenciones, como a llevar a cabo innovaciones en los tipos de actividades trabajadas en clase.

\section{Evaluación para la promoción de los estudiantes}

En uno de los espacios académicos observados se perciben estrategias de evaluación más en consonancia con las nuevas tendencias educativas para la evaluación del aprendizaje, puesto que hay una evaluación permanente insertada en el trabajo diario que se realiza en clase; se proponen así un gran número de actividades diversas para el aprendizaje y cuyo desarrollo se evalúa, como lo indica el profesor:

El proceso de aprendizaje será evaluado teniendo en cuenta los siguientes aspectos: trabajo y participación en clase, desempeño en la elaboración de talleres y trabajos propuestos, asistencia a clase y evaluaciones escritas, presentación y sustentación de actividades complementarias o de recuperación para quien no supere los logros propuestos. 
En este curso hay una total concordancia entre el trabajo que se hace en clase y lo que se evalúa. Todo esto posibilita que el profesor cuente con una cantidad importante de información proveniente de distintos trabajos de los estudiantes, y pueda hacer un seguimiento casi personal a cada uno, sobre sus avances y dificultades; para el estudiante abre diferentes oportunidades de demostrar su progreso y corregir sus errores; un estudiante se refiere a esto diciendo del profesor: "Tiene gran interés por los alumnos, se puede dedicar a cada persona el tiempo necesario para que supere las dudas que tiene". Sin demeritar el esfuerzo y el trabajo del profesor, ni restar relevancia a la innovación en la evaluación, y por el contrario destacando su pertinencia y validez, consideramos que la implementación de estas estrategias se facilita en gran parte por tratarse de un curso que corresponde al ambiente de tecnología de la Licenciatura en Matemáticas, cuyos propósitos atienden al aprendizaje de un contenido más técnico y ligado al sentido común, y donde el uso del computador como medio de trabajo que provee realimentación inmediata, se convierte en un colaborador determinante a la hora de valorar un trabajo como correcto o no.

Si bien, en otros dos de los espacios académicos observados la evaluación propuesta también está en concordancia con lo que se hace en clase, tanto desde el punto de vista del contenido que se valora como del tipo de tareas de evaluación que se proponen -quices, parciales, tareas, trabajos y exposiciones de éstos-, no se puede decir que en estos cursos haya innovación en la evaluación. Por ejemplo, el profesor dice: "Bueno, voy a dejar el ejercicio de tarea, la idea es que hagan más ejercicios para que apliquen las propiedades vistas", en consonancia con el trabajo de desarrollo de ejercicios que se ha hecho en clase; o en otra ocasión indica: "Saquen una hojita" y el profesor acepta que se desarrolle en parejas diciendo: "Bueno, háganse de a parejas, les voy a poner dos ejercicios y me los entregan resueltos". En uno de estos cursos, la excepción es una tarea consistente en elaborar un libro, que se desarrolla a lo largo de todo el semestre, la cual se evalúa al final, que podría constituirse en una tarea de evaluación diferente a las acostumbradas. Empero no hay información acerca de cómo se valoró ésta.

En el otro espacio académico, el profesor lleva a cabo una autoevaluación al final del semestre con la intención de compararla con la evaluación que él ha realizado sobre el trabajo de cada estudiante, en la que se tuvieron en cuenta, por lo menos de manera informal, los logros establecidos para el curso, como se puede apreciar en el siguiente fragmento de clase:

Profesor: Vamos a empezar. Estudiante $_{1}$

Estudiante $_{1}$ : Bueno, yo creo que B.

Profesor. ¿Por qué?

Estudiante $_{1}$ : Porque yo creo que... "Reconoce las invariantes conceptuales que identifican a un proyecto de aula en matemáticas", sí, pues no a profundidad, pero las trabajamos en la Unidad Didáctica, y creo que las demás las trabajamos...

Profesor: No, no, no. Venga les digo, para todos: cuando yo les pregunte, es tanto, no es uno por uno que me 
lo digan, sino en general: mire, yo considero que me merezco B porque es que no vine, sino solamente a dos clases y con esas dos participaciones yo soy casi genio...

En ocasiones este mismo profesor propone una actividad de coevaluación entre los estudiantes para las exposiciones de los trabajos de sus compañeros, en la cual también se consideraron los logros para el curso, y en una oportunidad, propone intercambiar entre los estudiantes los textos escritos por ellos para evaluarlos, pero al comunicar las valoraciones hechas, es claro que los estudiantes consideraron distintos aspectos para sus juicios, según se ve en el fragmento de clase que se expone a continuación:

Profesor: Socialicemos primero los trabajos y luego les digo lo que vamos a hacer.

Estudiante $_{2}:$ ¿Las correcciones que hicimos nosotros?

Profesor: Sí.

Estudiante $_{2}$ : Nosotros corregimos, bueno, hicimos anotaciones al trabajo de estudiante3, estudiante4 y estudiante $_{5}$.

Profesor: De los cuales hay dos de los tres.

Estudiante $_{2}$ : ¿El señor Estudiante? Bien, mucho gusto. Bueno, habían unas frases con las que teníamos problemas, por ejemplo, en la parte que dice: en esta Unidad Didáctica se presentan varias actividades escolares dirigidas a un grupo selecto de estudiantes, o sea, no entendemos por qué selecto...

En más de estas situaciones, en general en los espacios académicos, no se utilizan de manera frecuente, la au- toevaluación, la coevaluación, o técnicas de evaluación como los portafolios y los criterios de evaluación.

Para algunos de los objetivos, logros, indicadores de logro e intenciones desplegados en los programas de cada espacio académico, y a veces repetidos en clase por los profesores, no se registra que haya un seguimiento regular durante el semestre ni que se tengan en cuenta a la hora de evaluar; por ejemplo, es el caso de los logros relativos a la participación en clase, a la construcción social de conocimiento, o a otros objetivos como que "los estudiantes valoren la importancia del cálculo diferencial para su formación personal y futuro desempeño como docente". Esta situación se puede generalizar a los cuatro cursos, pero en uno de ellos sólo atañe a uno o dos logros, pues los demás logros exhibidos en el programa del curso, referidos a aprendizaje de comandos y desarrollo de destrezas sí se valoran en el desarrollo de las tareas.

Creemos que las dificultades para llevar a la práctica las nuevas visiones con respecto a la evaluación, están vinculadas a los problemas para innovar en la enseñanza y el aprendizaje, y mal podría un profesor que ha tratado un tema de cierta forma en sus clases, evaluarlo de otra. Por otra parte, sería deseable que los profesores evaluaran de manera más continua y que llevaran un registro más sistemático del progreso de los estudiantes que les permitiera hacer del proceso evaluativo una actividad más personalizada; sin embargo, este proceso, especialmente para asignaturas como matemáticas o de educación matemática, implica tiempo y dedicación, 
ambos recursos escasos en la profesión docente.

\section{Desarrollo del contenido}

Se puede percibir la afinidad entre lo previsto y lo hecho con relación al contenido tratado, pues los temas que se encuentran indicados en los programas de los cursos, se abordaron durante las clases. Esto no es tan claro, sin embargo, para uno de los espacios académicos observados, donde la resposabilidad de abordar las temáticas se deja a los estudiantes y en clase se tratan pocos de los tópicos correspondientes a la materia en cuestión. Se aprecia además que mientras en los otros tres espacios académicos se persigue una secuencia para el estudio de los temas mediante una trayectoria de cierta forma ya trazada, en este curso hay un derrotero marcado por el trabajo que los estudiantes van realizando sobre la elaboración de la unidad didáctica y que no es necesariamente secuencial pues las tareas implicadas están interrelacionadas y se requiere un ir y venir entre ellas. Aunque vemos el valor y la necesidad de la flexibilidad en el trabajo del maestro para ajustar la enseñanza a lo que está sucediendo en el aprendizaje de sus estudiantes, en este caso consideramos que hizo falta, al menos al comienzo del curso, un tratamiento de algunos de los tópicos requeridos para el desarrollo de dichas tareas.

Para unos de los espacios académicos observados se establecen propósitos y logros conceptuales más complejos de alcanzar que se refieren a que los estudiantes, por ejemplo, vean un concepto desde una perspectiva dada, interconecten conceptos, reconozcan invariantes conceptuales o avancen en el desarrollo de cierto tipo de pensamiento. "Aplicar el cálculo diferencial como herramienta para modelar situaciones de cambio y aproximación". "Estudiar las funciones desde la variación”. No obstante, las situaciones que se percibieron en clase encaminadas específicamente a tales fines, son contadas y no hay un seguimiento del progreso de los estudiantes en relación a esos logros. Cabría aquí exceptuar el espacio académico del ambiente de tecnología, donde a través del conjunto de tareas propuesto a los estudiantes pudo propiciarse el desarrollo de pensamiento lógico, aunque quizás de manera primitiva.

En todos los espacios académicos los estudiantes deben realizar lecturas; por ejemplo, un profesor indica: "Todas las lecturas son de carácter obligatorio. Leerlas... y esas lecturas las van a aplicar a su trabajo. Cada vez que lean algo, sean minuciosos". Sólo algunas de dichas lecturas se tratan después en clase, como en la situación siguiente donde el profesor dice: "Digamos lo que entendimos de la lectura”, “¿Quién quiere comenzar a contarnos sobre signos y símbolos?".

En el curso del ambiente de tecnología se proponen tareas para elaborar el mismo programa de computador a través de procesos invertidos, que aun sin ser del dominio matemático, podrían mirarse en un sentido de hacer y deshacer; sólo en pocos trabajos se involucraron las matemáticas. En otro espacio académico, de manera frecuente hay tareas realizadas en conjunto con el profesor que involucran reconocimiento de patrones, generalización de particularidades, formulación de conjeturas, 
como se puede ver en el siguiente fragmento de clase:

Profesor: :: Esos cuatro puntos pertenecen a un cuadrado. ¿Qué pasaría si se redujera el tamaño de los lados a la mitad?, ¿qué quedaría?

Estudiante: Un cuadrado.

Profesor: Y, ¿si sigo reduciéndolo?

Estudiante: Un punto.

Profesor: Entonces un punto, uno. Para obtener el siguiente, copio el anterior y agrego un punto al lado y otro encima de éste, pero como lo que se quería obtener era un cuadrado, se completa del otro lado dibujando tres puntos más, para por último contar nueve puntos.

$$
\text { i } \quad: \because \quad: \because::
$$

Continúen con el patrón. Ahora trabajen sobre los números triangulares.

En un curso distinto se trabaja bastante en la aplicación de procedimientos, como cuando se señala "utilizar la definición de límite para demostrar que el límite de la función $f(x)=2 x+1$ es 5 cuando $x$ tiende a 2". En el espacio académico de pedagogía cabe resaltar el énfasis que el profesor hace a través de algunas actividades, para que los estudiantes se den cuenta de que trabajar en pedagogía requiere profundizar en el estudio de las matemáticas. En algunas tareas específicas puede decirse que hubo lugar para algo de creatividad, en particular la tarea de elaborar un libro. Las indagaciones y exposiciones sobre la historia de las matemáticas pudieron contribuir con información nueva acerca de cómo se ha construido el conocimien- to matemático, como lo señalan unos estudiantes:

Para nosotros, excelente el desarrollar por distintos métodos lo que nosotros conocemos ya, sumas, divisiones, lo básico de la aritmética, y verlo cómo lo hacían hace, no sé, mil años, dos mil, tres mil, cinco mil... Nos hace que ver que hace doce mil años habían ya solucionado fracciones parciales, ya tenían áreas, un número muy cercano a pi, tenían cómo hallar áreas de pirámides, de triángulos, de rectángulos, de círculos.

El profesor corrobora estas apreciaciones:

Vieron la matemática metida, no solamente en una historia como algo que es divino, que apareció por obra y gracia del Espíritu Santo y entonces ya quedó descubierto, sino fueron unos problemas que los hicieron reflexionar y los orientaron.

Las tareas que pueden encuadrarse en la resolución de problemas, de acuerdo con su conceptualización en la teoría de la educación matemática, se dan con frecuencia sólo en uno de los espacios académicos de matemáticas, donde hay enunciados propuestos que a veces plantean problemas no rutinarios y lo suficientemente complejos para generar desafíos. En otro curso en los talleres del comienzo también existen algunos problemas que no implican encontrar un camino que no necesariamente se conoce pero no hay espacios para socializar ni discutir su solución. En dos de los cursos se rescata el intento por enmarcar el trabajo que se realizó, principalmente en las clases iniciales, 
dentro de contextos de la vida real; en uno de ellos con el planteamiento de situaciones cotidianas. El hecho de que, en ocasiones, los estudiantes presenten diferentes desarrollos o soluciones para la situación problemática planteada pudo dar lugar a la idea de respuestas no únicas en matemáticas.

Las posibilidades de que el trabajo que los estudiantes realizan en estos espacios académicos se llevara a cabo a partir de lo intuitivo, por medio de la exploración y el descubrimiento, con la socialización y comunicación necesarias, y de manera argumentada y justificada, fueron escasas. Hay al comienzo de un curso el desarrollo de unos talleres que apuntan en esta dirección, pero que luego no se vuelven a presentar por las dificultades metodológicas con los estudiantes, ya relatadas. En otro espacio académico, una experiencia en torno al desarrollo de una tarea en grupo con la finalidad de construir un sistema numérico, puede verse como una vivencia de lo que se denomina "hacer matemáticas", algunas actividades en este curso permiten explorar y justificar, pero son realizadas por los estudiantes en conjunto con el profesor, quien lleva la "batuta", como lo ilustra el siguiente fragmento de clase:

Profesor: (Escribe en el tablero)

$$
\begin{aligned}
\mathrm{T} 1 & =1 \\
\mathrm{~T} 2 & =3 \\
\mathrm{~T} 3 & =6 \\
\mathrm{~T} 4 & =10 \\
\mathrm{~T} 5 & =15
\end{aligned}
$$

Estudiante: Parece una sucesión.

Profesor: (Escribe en el tablero)

$$
\begin{aligned}
& \mathrm{T} 1=1 \\
& \mathrm{~T} 2=3=1+2=\mathrm{T} 1+2 \\
& \mathrm{~T} 3=6=3+3=\mathrm{T} 2+4 \\
& \mathrm{~T} 4=10=6+4=\mathrm{T} 3+5
\end{aligned}
$$

Profesor: ¿Cuál es la regularidad?

Estudiante: ...

Profesor: Se podría modelar con... Es una conjetura...

$$
\mathrm{T} n=n(n+1) / 2
$$

Profesor: Verifiquemos

$$
\begin{aligned}
& \mathrm{T} 5=\mathrm{T} 4+5 \\
& \mathrm{~T} 5=(\mathrm{T} 3+4)+5 \\
& \mathrm{~T} 5=\mathrm{T} 2+3+4+5 \\
& \mathrm{~T} 5=\mathrm{T} 1+2+3+4+5 \\
& \mathrm{~T} 5=1+2+3+4+5 \\
& \mathrm{~T} 5=15=5 \cdot 6 / 2 \\
& \mathrm{~T} 5=5 \cdot(5+1) / 2
\end{aligned}
$$

En los cursos a veces se hacen preguntas de por qué a las respuestas de los estudiantes, pero no son usuales; las justificaciones expuestas no se discuten y dado que no se aborda en qué consiste un argumento o justificación adecuada, se encuentra que éstas son de distintos estilos, según se aprecia en el siguiente fragmento de clase:

Profesor: (Escribe en el tablero)

$f(x)=x^{2}-5 x+6$ (Pregunta por la derivada de esa función); los estudiantes $\mathrm{E}: f^{\prime}(x)=2 x-5$

Profesor. Esta es la notación moderna de la derivada. Si igualamos a cero la derivada $x=5 / 2$. ¿Cuándo hay un máximo?

\section{Estudiante: ...}

Profesor: ¿Cómo se sabe si $5 / 2$ es un máximo, mínimo o un punto de inflexión? 
Estudiante: Yo tomaba el 5/2 y lo remplazaba en la función.

Profesor: (Escribe en el tablero)

$$
\begin{gathered}
\frac{25}{4}-\frac{25}{2}+6 \\
-\frac{25}{4}+\frac{24}{4}=-\frac{1}{4}
\end{gathered}
$$

Profesor: ¿Qué es -1/4?, ¿un máximo, un mínimo o un punto de inflexión?

\section{Estudiante: ...}

Profesor: A lo que voy es que hay que repasar los criterios de la primera y de la segunda derivada.

En uno de los espacios académicos hay alguna actividad relacionada con la traducción entre sistemas de representación matemática en sentidos no acostumbrados, de la gráfica a la representación algebraica y viceversa, sobre todo en las primeras tareas. En otro de los cursos hay un trabajo puntal en esta dirección, pero sólo en contadas ocasiones. Curiosamente, en el espacio académico del ambiente de tecnología podría hablarse de manejo de distintas representaciones, obviamente no matemáticas, y de traducción entre ellas en todos los sentidos. No obstante, en general no se percibe un énfasis en el trabajo de traducción entre representaciones.

La utilización de recursos tecnológicos se da en un curso con el uso de la calculadora simplemente para llevar a cabo cálculos, y en el curso de tecnología con el empleo del computador, como herramienta indispensable para el trabajo, que seguramente contribuyó a que los estudiantes se motivaran a trabajar.

En síntesis, el tratamiento didáctico para el desarrollo del contenido matemático en los cursos observados, presenta algunos elementos distintos a los usuales, pero no coherentemente hilados ni reiterados para que, por ejemplo, los estudiantes tengan en clase suficientes vivencias de lo que puede significar "hacer matemáticas", "resolver problemas", traducir en todos los sentidos entre diversos sistemas de representación, aproximarse a los conceptos desde distintos ángulos, trabajar en contextos de la vida real, proponer diferentes estrategias y soluciones válidas, comunicar ideas en lenguaje natural, justificar respuestas con base en argumentos matemáticos, aprovechar la tecnología para el desarrollo de matemáticas. En consecuencia, podría decirse, que es poco el aporte de estos cursos a una transformación de la visión de los estudiantes sobre lo que son las matemáticas, su enseñanza y aprendizaje.

\section{Anotaciones finales}

Es claro que existen diferencias entre las rutas pedagógicas seguidas en los espacios académicos que se observaron. Las distinciones, que conciernen en especial a algunos de los aspectos considerados, están sin duda determinadas por la naturaleza del conocimiento que se aborda en cada curso y están ligadas de manera obvia al profesor y a sus visiones, su conocimiento y experiencia. Como lo expresan los profesores en las entrevistas, algunas de las rutas seguidas en clase nacen de su convencimiento del posible beneficio para los estudiantes. Quizás también estas rutas son vistas por los profesores como el camino para alcanzar los logros e intenciones manifestadas al comienzo del semestre y les parece que a través de ellas se implementan de manera adecuada las teorías y constructos que se manejan en la comunidad educativa y 
que han incluido en el planteamiento de dichos logros.

No obstante lo anterior, y a pesar de que las rutas pedagógicas, en algunos de sus aspectos, se pueden destacar como variantes a las que surgen por razón del quehacer docente tradicional, y posiblemente contribuyen a la formación de los futuros maestros, creemos que hace falta una reflexión más cuidadosa de parte de los profesores en torno a las nuevas teorías y propuestas, a su significado y a su implementación, con el propósito de que la innovación vaya más allá del nivel del vocabulario, y se evidencie una transformación real en las rutas seguidas. En particular esto es cierto, con respecto a expresiones como construcción social del conocimiento, formación ciudadana, desarrollo de pensamiento matemático. Es decir, se requiere que el diseño y desarrollo curricular específico de los temas a tratar, contemple, además de intenciones, las actividades y tareas que se van a trabajar para aterrizarlas

\section{Bibliografía}

Andrade, L. y Perry, P. (2002). La formación de profesores: un reto con muchos interrogantes. Epsilón, 52, 147-172.

Andrade, L.; Perry, P.; Guacaneme, E. y Fernández, F. (2003a). Rutas pedagógicas en matemáticas: ¿azar o construcción? Bogotá: IDEP y Una empresa docente.

(2003b). La enseñanza de las matemáticas: ¿en camino de transformación? Relime, 6(2), 81-105.

Beltrán, J.; Pardo, J. y Cruz, J. (2003). Mapas, redes y horizontes: el concepto de ruta pedagógica. Aula Urbana, $45,10-11$. y alcanzarlas, y los mecanismos de observación y seguimiento a su evolución. Es imperativo igualmente, desarrollar la capacidad de mirarse y la conciencia de lo que se hace habitualmente en el salón de clase, con el fin de que los caminos que se tomen hayan sido madurados, pues como señala Mason (1996), la sobrecarga cognitiva implicada en la labor de enseñar conduce a que usualmente lo que se hace en clase sea simplemente reaccionar, para manejar y despachar los asuntos que surgen, de la mejor forma posible.

Aunque se percibieron algunas innovaciones en las rutas propuestas para que los estudiantes se aproximen y trabajen el conocimiento disciplinar, fue evidente que en dos de los cursos hubo que volver a una trayectoria usual ya que las rutas carecieron de una planeación y previsión seria, que permitiera retomarlas en caso de dificultades y lograr una incidencia real en el aprendizaje de los estudiantes. $\boldsymbol{\Delta}$

Bishop, A., y Clarkson, P. C. (1988). What values do you think you are teaching when you teach mathematics? En: Gough, J. y Mousley, J. (eds.). Exploring all angles. Melbourne: Mathematics Association of Victoria, pp. 30-38.

Bonilla, M. y Romero, J. (2000). A propósito del saber del profesor de matemáticas. Revista EMA, 5(2), 189-191.

Cantoral, R. (2001). Conocimiento matemático y su construcción social. Documento de trabajo. México: CINVESTAV. 
Carpenter, T.; Fenema E. y Franken, M. (1997). Instrucción guiada cognitivamente: una base para la reforma de la enseñanza de matemáticas en primaria. Revista EMA, 3(1), 3-32.

Christiansen, I. (1999). Reflexiones críticas sobre modelos matemáticos en la clase: ¿sueño o realidad? Revista EMA, 5(1), 29-50.

Cobb, P. y McClain, K. (2001). Supporting students' learning of significant mathematical ideas. Documento de trabajo investigativo.

Cobb, P. y Yackel, E. (1996). Constructivist, emergent and sociocultural perspectives in the context of developmental research. Educational Psychologist, 31, 175-190.

Cockcroft, W.H. (1985). Las matemáticas sí cuentan (Informe Cockcroft). Madrid: Ministerio de Educación y Ciencia.

De Lange, J. (1995). Assessment: No change without problems. En: Romberg, T. (ed.). Reform in school mathematics and authentic assessment. Albany: State University of New York Press, pp. 87-172.

Denzin, N. y Lincoln, Y. (1998). Entering the field of qualitative research. En: Denzin, N. y Lincoln, Y. (eds.). Strategies of qualitative inquiry. Thousand Oaks, CA.: Sage Publications, Inc, pp. 1-34.

Departamento de Matemáticas-Universidad Pedagógica Nacional. (2002). Referentes del proyecto curricular Licenciatura en Matemáticas. Condiciones iniciales. Documento institucional. Bogotá: Universidad Pedagógica Nacional

Doyle, W. (1986). Classroom organization and management. En: Wittrock, M.C. (ed.). Handbook of research on teaching. Nueva York: Macmillan $\mathrm{Pu}-$ blishing Company, pp. 392-431.

Eisenhart, M. (1988). The etnographic research tradition and mathematics education research. Journal for Research in Mathematics Education, 19(2), 99-114.

Jaworski, B. (1994). Investigating mathematics teaching. A constructivist enquire. London: The Falmer Press.

Kilpatrick, J. (1995). Técnicas de evaluación para profesores de matemáticas de secundaria. En: Kilpatrick, J.; Gómez, P. y Rico, L. (eds.). Educación Matemática. Bogotá: Una empresa docente.

Llinares, S. (1994). El profesor de matemáticas. Conocimiento base para la enseñanza y desarrollo profesional. En: Santaló, L. A.; Llinares, S.; Sánchez, V.; Taibo, A. y García-Hoz, A. (eds.). La enseñanza de las matemáticas en la educación intermedia. Madrid: Rialp, pp. 296-340.

Mason, J. (1996). Personal enquiry: Moving from concern towards research. United Kingdom: The Open University.

Moreno, L. (1997). La educación matemática hoy. Revista EMA, 2(2), 101-114.

National Council of Teachers of Mathematics. (1989). Curriculum and evaluation standards for school mathematics. Reston, VA: National Council of Teachers of Mathematics.

(1991). Professional standards for teaching mathematics. Reston, VA: National Council of Teachers of Mathematics.

(2000). Principles and standards for school mathematics. Reston, VA: National Council of Teachers of Mathematics. 
Ponte, J. P.; Boavida, A. M.; Graça, M. y Abrantes, P. (1997). Funcionamiento de la clase de matemáticas. En: Didáctica da matemática. Lisboa: Ministerio de Educaçao, PRODEP, pp. 71-95.

Pólya, G. (1957). How to solve it. Princeton, New Jersey: Princeton University Press.

Quinn, M. (2002). Qualitative research and evaluation methods. Thousand Oaks, CA.: Sage Publications, Inc.

Rico, L. (1990). Diseño curricular en Educación Matemática. Elementos y evaluación. En: Llenares, S. y Sánchez, V. (eds.). Teoría y práctica en Educación Matemática. Sevilla: Ediciones Alfar, pp. 117-171.

. (1995). Consideraciones sobre el currículo escolar de matemáticas. Revista EMA, 1(1), 4-24.

. (1997). Dimensiones y componentes de la noción de currículo. En: Rico, L. (ed.). Bases teóricas del currículo de matemáticas en educación secundaria. Madrid: Síntesis.

Romberg, T. (1991). Características problemáticas del currículo escolar de matemáticas. Revista de Educación, 294, 323-406.

Sfard, A. (1996). On acquisition metaphor and participation metaphor for the mathematics learning. En: Alsina, C.; Álvarez, J. M.; Hodgson, B.; Laborde, C. y Pérez, A. (eds.). 8th International Congress on Mathematical Education. Selected Lectures.

(1998). On two metaphors for learning and the dangers of choosing just one. Documento de trabajo.

. (2000a). On reform movement and the limits of mathematical discourse.
Mathematical Thinking and Learning, 2(3), 157-189.

(2000b). Symbolizing mathematical reality into being-or how mathematical discourse and mathematical objects create each other. En: Cobb, P.; Yackel, E. y McClain, K. (eds.). Symbolizing and communicating in mathematics classrooms. Mahwah, NJ.: Lawrence Erlbaum Associates Inc., Publishers, pp. 37-98.

(2001). There is more to discourse than meets the ears: Looking at thinking as communicating to learn more about mathematical learning. Educational Studies in Mathematics, 46(1/3), 13-57.

Skovsmose, O. (1999). Hacia una filosofía de la educación matemática crítica. Bogotá: Una empresa docente. (2000). Escenarios de investigación. Revista EMA, 6(1), 3-26.

Waldegg, G. (1998). Principios constructivistas para la eduación matemática. Revista EMA, 4(1), 16-31.

Yackel, E. (2000). Introduction: Perspectives on semiotics and instruccional design. En: Cobb, P.; Yackel, E. y McClain, K. (eds.). Symbolizing and communicating in mathematics classrooms. Mahwah, NJ.: Lawrence Erlbaum Associates Inc., Publishers, pp. 1-13.

Yackel, E. y Cobb, P. (1996). Sociomathematical norms, argumentation, and autonomy in matehmatics. Journal for Research in Mathematics Education, 27(4), 458-477. 